\title{
Aprotinin improves kidney function and decreases tubular cell apoptosis and proapoptotic signaling after renal ischemia-reperfusion
}

Ajay Kher, MD, ${ }^{a}$ Kirstan K. Meldrum, MD, ${ }^{b}$ Karen L. Hile, BS, ${ }^{b}$ Meijing Wang, MD, ${ }^{a, d}$ Ben M. Tsai, MD, ${ }^{a}$ Mark W. Turrentine, MD, ${ }^{a}$ John W. Brown, MD, and Daniel R. Meldrum, MD ${ }^{a, c, d, e}$

Extra illustrations are available online. $\bigcirc$
From the Departments of Surgery, ${ }^{\mathrm{a}}$ Urology, , and Cellular and Integrative Physiology, ${ }^{\mathrm{c}}$ the Indiana Center for Vascular Biology and Medicine, ${ }^{\mathrm{d}}$ and the Center for Immunobiology, ${ }^{\mathrm{e}}$ Indiana University Medical Center, Indianapolis, Ind.

Supported in part by National Institutes of Health grant R01GM070628 (D.R.M.), the Clarian Values Fund (D.R.M.), the Showalter Trust (D.R.M.), the Cryptic Masons Medical Research Foundation (D.R.M., M.W.), and a phase IV grant from Bayer Pharmaceuticals Corporation.

Received for publication Oct 26, 2004; revision received Feb 8, 2005; accepted for publication Feb 15, 2005

Address for reprints: Daniel R. Meldrum, MD, 545 Barnhill Dr, Emerson Hall 215, Indianapolis, IN 46202 (E-mail: dmeldrum@ iupui.edu).

J Thorac Cardiovasc Surg 2005;130:662-9

$0022-5223 / \$ 30.00$

Copyright (C) 2005 by The American Association for Thoracic Surgery

doi:10.1016/j.jtcvs.2005.02.035
Objective: The purpose of the study was to determine the effects of aprotinin on (1) renal function, (2) apoptosis and apoptotic signaling, and (3) the inflammatory response of the kidney after ischemia-reperfusion injury.

Methods: Male rats underwent a sham procedure or left renal ischemia for 1 hour. Rats were divided into three groups and received no reperfusion, reperfusion for 1 hour, or reperfusion for 24 hours. The animals undergoing ischemia received saline solution alone or aprotinin $(60,000 \mathrm{kIU} / \mathrm{kg})$. At the end of the experiment, a sample for serum creatinine was taken and the left kidney was harvested. The kidney was analyzed for expression of tumor necrosis factor $\alpha$, interleukin $1 \beta$, and interleukin 6 (enzyme-linked immunosorbent assay and reverse transcriptase-polymerase chain reaction) and activation of p38 mitogen-activated protein kinase, caspase 3, and caspase 8 (Western blot). The kidney was assessed for apoptosis with enzymelinked immunosorbent assay and by terminal deoxynucleotidyl transferase biotindeoxyuridine triphosphate nick-end labeling staining of tissue slides.

Results: Aprotinin significantly decreased the rise in serum creatinine and apoptosis caused by ischemia-reperfusion. Aprotinin significantly reduced interleukin 1 and 6 messenger RNA production and showed a trend toward reducing tumor necrosis factor messenger RNA production after ischemia. Aprotinin also significantly reduced caspase 8 activation and showed a trend toward decreasing p38 mitogenactivated protein kinase activation after 1 hour of reperfusion.

Conclusion: These results suggest that aprotinin provides protection from renal ischemia-reperfusion injury. They also suggest that aprotinin may do so by affecting apoptotic signaling and inflammatory cytokine production. Aprotinin is a potential therapeutic measure in clinical situations where renal ischemia-reperfusion injury can be anticipated, provided adequate heparinization is possible.

$\mathrm{R}$ enal ischemia-reperfusion leads to injury through many mechanisms, such as calcium dyshomeostasis, oxygen free radical production, mitochondrial dysfunction, cytokine generation, and neutrophil sequestration and activation. The role of inflammation in this injury is being increasingly delineated. ${ }^{1-9}$ Aprotinin, a serine protease inhibitor, inhibits many enzymes, including those involved in coagulation, fibrinolysis, and inflammation. Aprotinin has been used to improve hemostasis and reduce the blood transfusion requirements in cardiac surgery. Aprotinin has anti-inflammatory properties and reduces the injury produced by leukocytes by inhibiting leukocyte hyperactivation, ${ }^{10}$ leukocyte integrin expression, ${ }^{11,12}$ and leukocyte extravasation. ${ }^{13}$ Aprotinin has also been shown to decrease in vitro tumor necrosis factor (TNF) $\alpha$ production by macrophages, ${ }^{14}$ decrease plasma TNF- $\alpha$ levels in patients undergoing coronary artery bypass grafting, ${ }^{15}$ and 
suppress the release and activity of TNF- $\alpha$ in rat hearts undergoing cold storage. ${ }^{16}$ Aprotinin also has been seen to decrease the production of interleukin (IL) $6^{17}$ while increasing the production of anti-inflammatory IL-10 in cardiac surgical patients. ${ }^{18}$

Concern regarding the use of aprotinin has been raised because it has been reported to increase renal dysfunction. ${ }^{19}$ Studies showed a significant number of patients to have a greater than $0.5 \mathrm{mg} / \mathrm{dL}$ increase in serum creatinine with aprotinin, but the increase was transient and did not lead to clinically significant renal disease. ${ }^{20,21}$ Other studies and a recent meta-analysis have shown no difference between aprotinin and placebo in the incidence of renal failure after coronary artery bypass grafting. ${ }^{22-24}$

Ischemia-reperfusion injury can lead to cell death by necrosis or apoptosis. Prolonged renal ischemia leads to necrotic cell death, whereas in animal models with shorter periods of renal ischemia, apoptosis is the primary mode of cell death. ${ }^{25}$ Aprotinin has been shown to reduce cardiomyocyte apoptosis after myocardial ischemia-reperfusion injury, ${ }^{26}$ suggesting the possibility of a similar effect in renal ischemiareperfusion.

The purposes of the study were to (1) determine the effect of aprotinin on renal function after ischemia-reperfusion injury, (2) examine the effects of aprotinin on apoptosis and apoptotic signaling after ischemia-reperfusion injury, and (3) determine the effects of aprotinin on the inflammatory response of the kidney to ischemia-reperfusion injury.

\section{Materials and Methods Animals}

Male Sprague-Dawley rats weighing 250 to $300 \mathrm{~g}$ (Harlan Sprague Dawley, Inc, Indianapolis, Ind) were acclimated and maintained on a standard pellet diet for 1 week before the initiation of experiments. Animals were anesthetized with isoflurane. The animal protocol was reviewed and approved by the Indiana Animal Care and Use Committee of Indiana University. All animals received humane care in compliance with the "Guide for the Care and Use of Laboratory Animals" (http://www.nap.edu/catalog/5140.html).

\section{Experimental Groups and Operative Techniques}

Animals were divided into the following experimental groups: (1) 1-hour sham controls $(n=5),(2) 2$-hour sham controls $(n=5)$, (3) 25-hour sham controls $(\mathrm{n}=5)$, (4) 1-hour ischemia $(\mathrm{n}=12)$, (5) 1-hour ischemia and 1-hour reperfusion $(\mathrm{n}=12)$, and (6) 1 -hour ischemia and 24-hour reperfusion $(n=12)$. Animals undergoing ischemia were further allocated to receive either aprotinin at $60,000 \mathrm{kIU} / \mathrm{kg}$ or an equivalent volume of saline solution through tail vein injection 15 minutes before initiation of ischemia. Twenty-five hour sham controls (group 3) and animals undergoing 24-hour reperfusion (group 6) also received $1200 \mathrm{U} / \mathrm{kg}$ heparin subcutaneously 30 minutes before laparotomy and underwent nephrectomy of the right kidney. After induction of anesthesia, the left renal pedicle was isolated through a midline laparotomy and occluded with an atraumatic snare. The abdomen was subsequently closed. The animals were allowed to awaken spontaneously. In the reperfusion groups, the snare was removed externally without need for abdominal reentry. The sham controls underwent the same procedure without occlusion of the renal pedicle. On completion of the experiment, the animals were anesthetized, serum samples were taken, and the left kidney was removed. A portion of the kidney was kept in $30 \%$ sucrose at $4^{\circ} \mathrm{C}$ for staining for apoptosis, and the remainder was frozen in liquid nitrogen. The tissue was stored at $-70^{\circ} \mathrm{C}$ until further testing could be performed. All animals were humanely killed.

\section{Renal Function Analysis}

To avoid the confounding, compensatory creatinine clearance of the contralateral kidney, rats undergoing reperfusion for 24 hours and the 25-hour sham controls underwent right nephrectomy at the same time as the left renal ischemia. Serum for creatinine measurement was taken at the end of reperfusion.

\section{Apoptosis}

Apoptosis in the kidney was assessed with a commercially available enzyme-linked immunosorbent assay (ELISA) kit (Cell Death Detection ELISAPLUS; Roche Diagnostics Corporation, Laboratory Systems, Indianapolis, Ind) that detects mononucleosomes and oligonucleosomes. ELISA was performed according to the manufacturer's instructions. Results are depicted as enrichment factor, the ratio of the sample value (in milliunits) to the negative control (sham controls) value (also in milliunits). The tissue was also examined for apoptosis with a commercially available kit (DeadEnd Fluorimetric TUNEL System; Promega Corporation, Madison, Wis). The kit is based on terminal deoxynucleotidyl transferase incorporation of fluorescein-deoxyuridine triphosphate for detecting DNA strand breaks in the nuclei of cells undergoing apoptosis. The cell nuclei were then counterstained with bisbenzimide to ensure constant cell density across all treatment groups examined.

\section{Western Blotting}

Western blot analysis was performed to measure p38 mitogenactivated protein kinase (MAPK), caspase-3, and caspase- 8 . Kidney tissue was homogenized in cold buffer containing $20-\mathrm{mmol} / \mathrm{L}$ tris(hydroxymethyl)aminomethane ( $\mathrm{pH} 7.5), 150-\mathrm{mmol} / \mathrm{L}$ sodium chloride, 1-mmol/L ethylenediaminetetraacetic acid, 1-mmol/L ethyleneglycol-bis-( $\beta$-aminoethylether)- $\mathrm{N}, \mathrm{N}, \mathrm{N}^{\prime}, \mathrm{N}^{\prime}$-tetraacetic acid, $1 \%$ Triton X-100 (Sigma-Aldrich Corporation, St Louis, Mo), $2.5-\mathrm{mmol} / \mathrm{L}$ sodium pyrophosphate, $1-\mathrm{mmol} / \mathrm{L} \beta$-glycerophosphate, $1-\mathrm{mmol} / \mathrm{L}$ sodium vanadate, $1-\mu \mathrm{g} / \mathrm{mL}$ leupeptin, and 1-mmol/L phenyl methyl sulfonyl fluoride and centrifuged at $12,000 \mathrm{rpm}$ for 5 minutes. The protein extracts $(50 \mu \mathrm{g} / \mathrm{lane})$ were subjected to electrophoresis on a $12 \%$ tris(hydroxymethyl)aminomethane hydrochloride gel (Bio-Rad Laboratories Inc, Hercules, Calif) and transferred to a nitrocellulose membrane, which was stained with naphthol blue-black to confirm equal protein loading. The membranes were incubated in 5\% dry milk for 1 hour and then incubated with the following primary antibodies: p38 MAPK and phospho-p38 MAPK (Thr180/Tyr182) antibody (Cell Signaling Technology, Beverly, Mass) and caspase 3 (H-277) and 8 (H-134) antibodies (Santa Cruz Biotechnology, Inc, Santa Cruz, Calif), followed by incubation with horseradish peroxidase-conjugated 


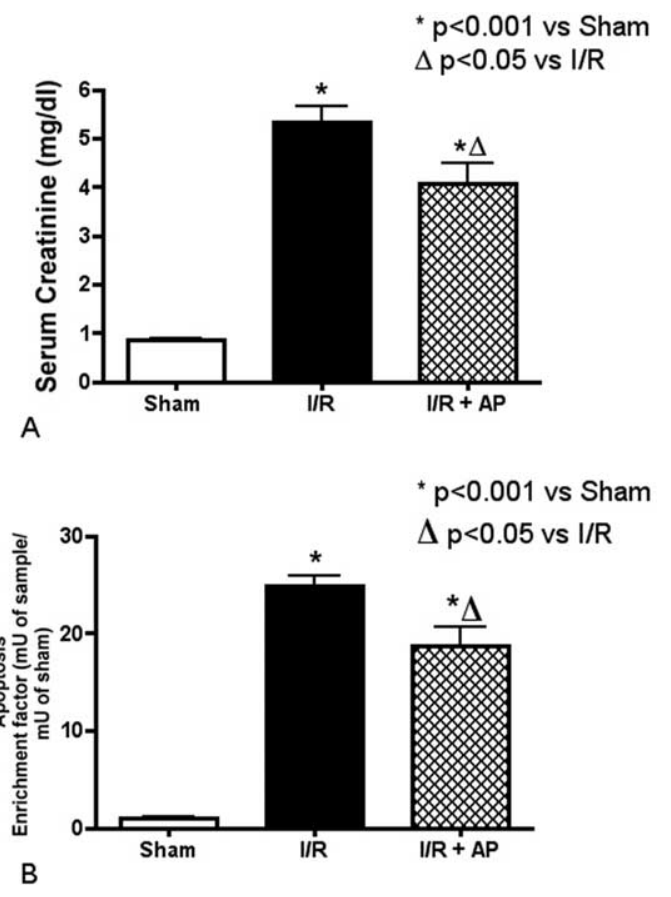

Figure 1. Renal function and apoptosis after 24 hours of reperfusion. A, Serum creatinine levels in 25 -hour sham controls (Sham), 1 hour of ischemia and 24 hours of reperfusion with saline solution $(I / R)$, and 1 hour of ischemia and 24 hours of reperfusion with aprotinin $(I / R+A P)$. B, Apoptosis ELISA in 25-hour sham controls (Sham), 1 hour of ischemia and 24 hours of reperfusion with saline solution $(I / R)$, and 1 hour of ischemia and 24 hours of reperfusion with aprotinin $(I / R+A P)$. Results are depicted as enrichment factor, ratio of sample value to negative control (sham controls) value. Bars represent mean; error bars indicate SEM. Asterisk indicates $P<.001$ relative to sham controls; delta indicates $\boldsymbol{P}<.05$ relative to ischemia-reperfusion with saline solution.

goat antirabbit immunoglobulin G secondary antibody and detection with Supersignal West Pico Stable Peroxide Solution (Pierce Chemical Company, Rockford, Ill). Films were scanned with an Epson Perfection 3200 Scanner (Epson America, Inc, Long Beach, Calif), and band density was analyzed with TotalLab software (Nonlinear Dynamics Ltd, Newcastle upon Tyne, United Kingdom).

\section{Reverse Transcriptase-Polymerase Chain Reaction}

Total RNA was extracted from the kidney with RNA STAT-60 (Tel-Test, Inc, Friendswood, Tex). A 1.0- $\mu$ g sample of total RNA was subjected to complementary DNA (cDNA) synthesis with cloned AMV first-strand cDNA synthesis kit (Invitrogen Corporation, Carlsbad, Calif). The cDNA from each sample was used for TNF- $\alpha$, IL-1 $\beta$, and IL-6 polymerase chain reaction (PCR) with dual quantitative reverse transcriptase (RT) PCR kits (Maxim Biotech, Inc, South San Francisco, Calif). One negative control used double-distilled water instead of RNA sample, and a second negative control used double-distilled water instead of RT, to exclude the presence of genomic contaminants. Positive controls were included in the kit to verify appropriate expression of respective markers. PCR products were separated by electrophoresis on $1 \%$ agarose gel stained with ethidium bromide. Representative gel photographs are shown, and densitometry was performed to assess relative quantity, represented as a ratio to reduced glyceraldehydephosphate dehydrogenase (GAPDH).

\section{Renal TNF- $\alpha$, IL-1 $\beta$, and IL-6}

Commercially available ELISA kits were used to determine kidney homogenate TNF- $\alpha$, IL- $1 \beta$ and IL-6 content. TNF- $\alpha$ and IL-6 contents were determined by BD OptEIA rat TNF ELISA set and BD OptEIA rat IL-6 ELISA set (BD Biosciences, San Jose, Calif). IL- $1 \beta$ was determined by Rat IL-1 $\beta /$ IL-1F2 Duo set (R\&D Systems, Minneapolis, Minn). ELISA was performed by adding 100 $\mu \mathrm{L}$ of each sample (equal protein and tested in duplicate) to wells in a 96-well plate. ELISA was performed according to the manufacturer's instructions. Final results are expressed as picograms of respective cytokine per milligram of protein.

\section{Statistical Analysis}

Data are presented as mean \pm SEM ( $n=5$ to 6 animals in each group). The experimental groups were compared by analysis of variance with a post hoc Bonferroni-Dunn test and unpaired $t$ tests.

\section{Results}

\section{Renal Function}

Serum creatinine was measured from uninephrectomized rats (nephrectomy was performed at the same time as the ischemia) at the end of 24 hours of reperfusion. Ischemiareperfusion resulted in marked renal damage, as demonstrated by the increase in serum creatinine. Administration of aprotinin resulted in a significant reduction in serum creatinine (Figure 1, $A$ ).

\section{Apoptosis}

Apoptosis was assessed at the end of 24 hours of reperfusion. Ischemia-reperfusion led to increased apoptosis, as assessed by ELISA, which was significantly decreased by the administration of aprotinin (Figure 1,B). Apoptosis was also assessed by fluorescein TUNEL staining of the kidney, which corroborated the findings of ELISA (Figure 2).

\section{Renal Caspase Expression After Ischemia-Reperfusion Injury}

The expression of apoptosis-related caspases in ischemiareperfusion-injured kidney was assessed by Western blotting. Caspase 8 expression was significantly higher after ischemia, but aprotinin did not affect it. After 1 hour of reperfusion, aprotinin significantly reduced caspase 8 expression (Figure 3, $A$ and $B$ ). However, no difference was observed in the expression of caspase 3 after ischemia or 1 hour of reperfusion relative to sham controls, and use of aprotinin did not affect the response (Figure 3, $C$ and $D$ ). 

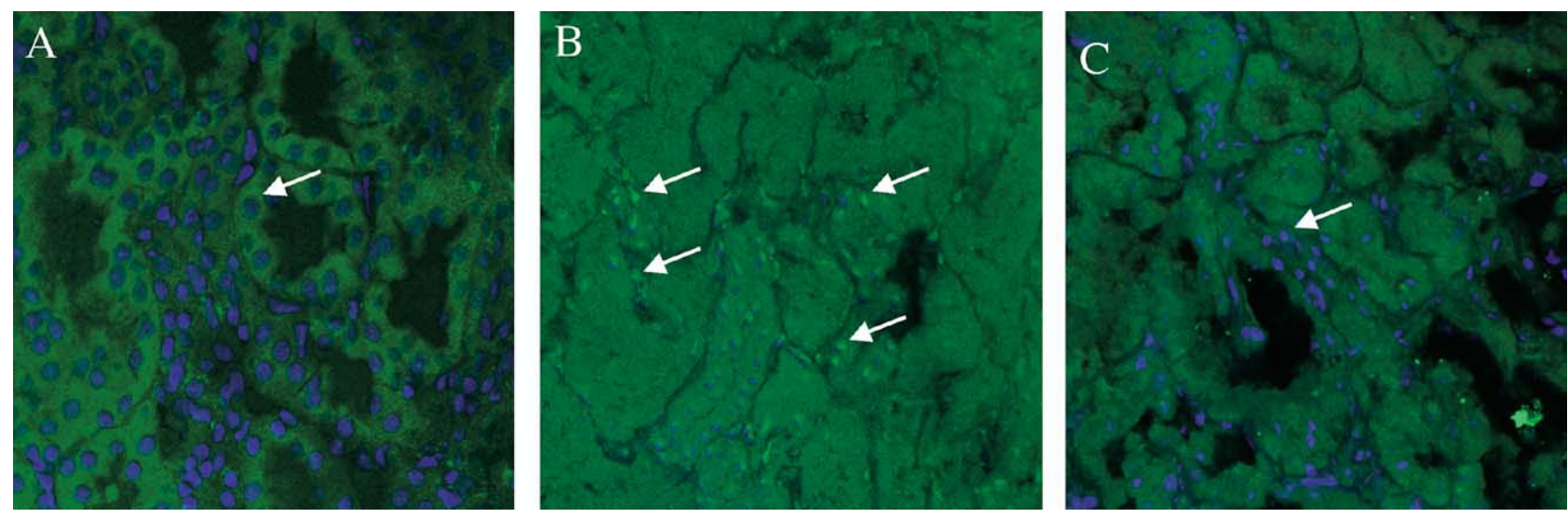

Figure 2. Photomicrographs (original magnification $200 \times$ ) demonstrating renal apoptosis. Nuclear stain (bisbenzimide, blue) shows nonapoptotic cells; apoptosis stain (TUNEL assay, green) shows apoptotic cells. A, In 25-hour sham control, white arrow points to nonapoptotic cell. B, In ischemia-reperfusion with saline solution, white arrows point to apoptotic cells. C, In ischemia-reperfusion with aprotinin, white arrow points to nonapoptotic cell.

\section{p38 MAPK Activation}

Ischemia and reperfusion caused activation of p38 MAPK. Aprotinin did not affect the activation caused by ischemia but did show a trend toward decreased p38 MAPK activation after 1 hour of reperfusion (Figure 4).

\section{Expression of TNF- $\alpha$, IL-1 $\beta$, and IL-6}

ELISA and RT-PCR were used to assess the renal expressions of TNF- $\alpha$, IL- $1 \beta$, and IL-6. Aprotinin caused a significant reduction in the induction of IL- $1 \beta$ and IL- 6 messenger RNA (mRNA) caused by ischemia and showed a trend toward decreased TNF mRNA production (Figure 5). At the end of ischemia, no increase in cytokine protein expression was observed (Figure E1). After 1 hour of reperfusion, aprotinin did not affect the induction of cytokine mRNA (Figure E2) or cytokine protein expression (Figure E3).

\section{Discussion}

The results of this study represent an initial demonstration that aprotinin improves renal function and decreases ischemiareperfusion-induced apoptosis and apoptotic signaling after renal ischemia-reperfusion injury. In contrast to the increased serum creatinine that some clinical studies have reported with aprotinin use in cardiac surgical patients, our results show that aprotinin decreases serum creatinine after renal ischemia-reperfusion injury. Adequate heparinization plays an important role in preventing renal dysfunction after cardiac surgery, and although most studies on aprotinin have used standardized protocols for heparin administration, they have not provided data on clotting time. Many have postulated that aprotinin reduces the inflammation and fluid overload associated with cardiopulmonary bypass surgery, therefore relative to surgical controls, postoperative serum creatinine levels are more concentrated in patients receiving aprotinin. Another possible reason for the transient increase in creatinine caused by aprotinin may be the filtering and reabsorption of aprotinin in the kidney. The high dose of aprotinin might "overload" the kidney and cause the increase in creatinine. ${ }^{19,23}$ In our study, the kidney underwent ischemia-reperfusion injury, and it may be aprotinin's anti-inflammatory properties that acted to provide protection. Further support for the protective effects of aprotinin in renal ischemia-reperfusion injury is provided by the decreases in apoptosis and caspase 8 . The decrease in apoptosis that we observed is similar to the result obtained by Pruefer and colleagues ${ }^{26}$ in the heart after regional ischemia-reperfusion. Ozer and associates ${ }^{27}$ showed decreased epithelial necrosis and decreased inducible nitric oxide synthase expression with aprotinin use after renal ischemiareperfusion injury. However, they did not assess renal function, apoptosis, or renal inflammatory cytokine production.

The mechanism by which aprotinin mediates these protective effects is unclear. Aprotinin significantly reduced the induction of IL- $1 \beta$ and IL- 6 mRNA caused by ischemia. It also led to a decrease in p38 MAPK activation after reperfusion. This indicates that aprotinin may reduce proinflammatory cytokine production, as has been shown in other animal and clinical studies. ${ }^{14-18}$ However, we were not able to find any difference with the use of aprotinin in the expression of cytokine protein. This may mean that the effect of aprotinin on the expression of cytokine proteins takes longer to manifest. It also may mean that aprotinin 


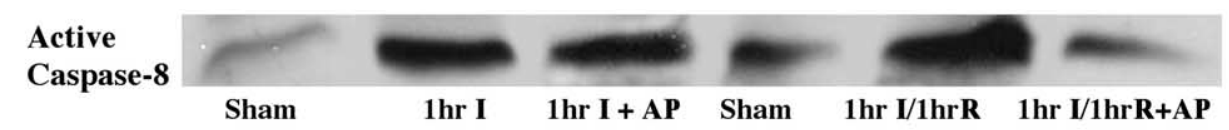

A
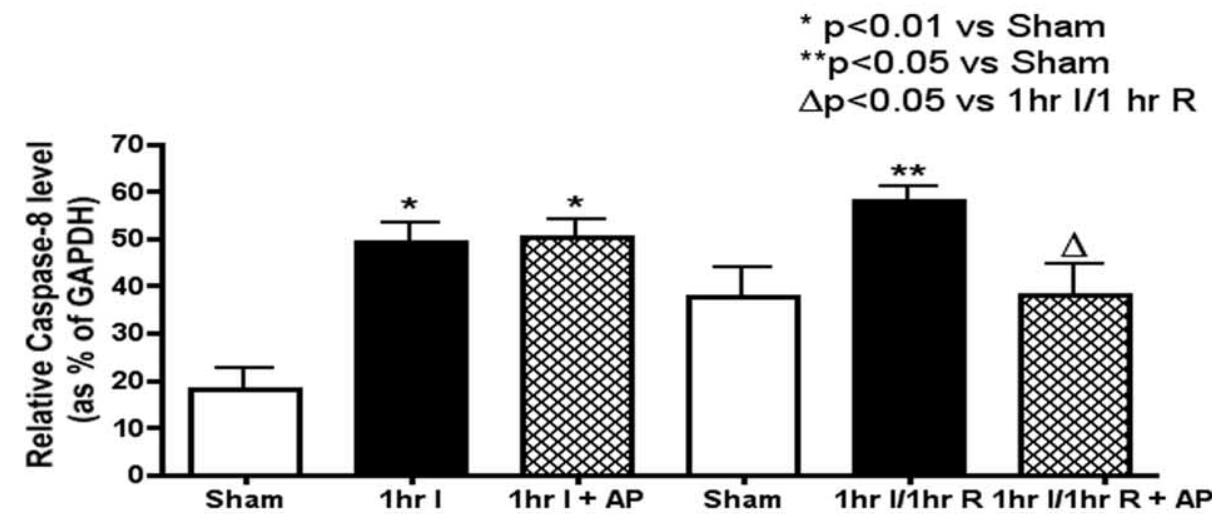

B

Active

Caspase-8

Sham

1hr I

I AP Sham

Ihr I/IhrR+AP
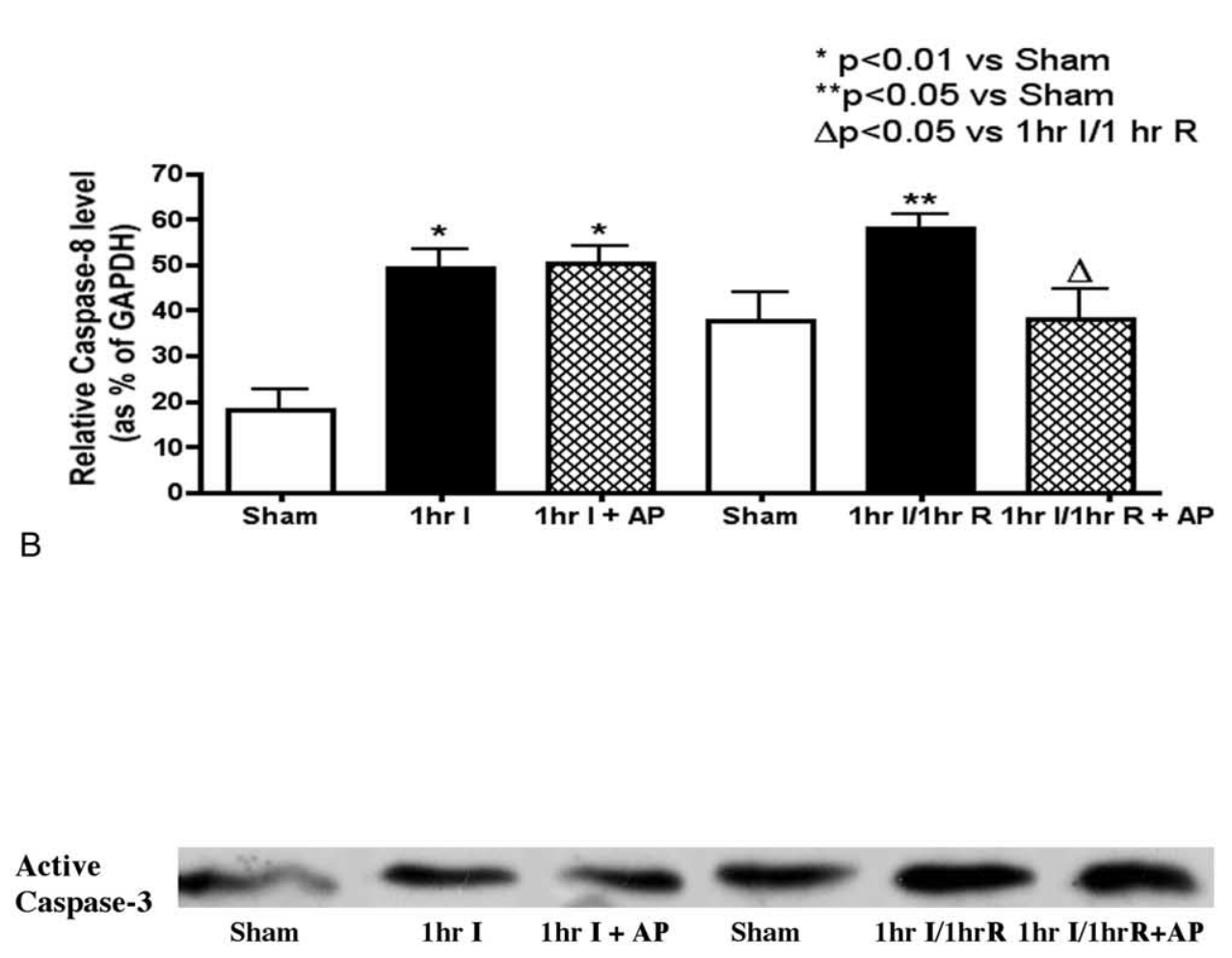

C

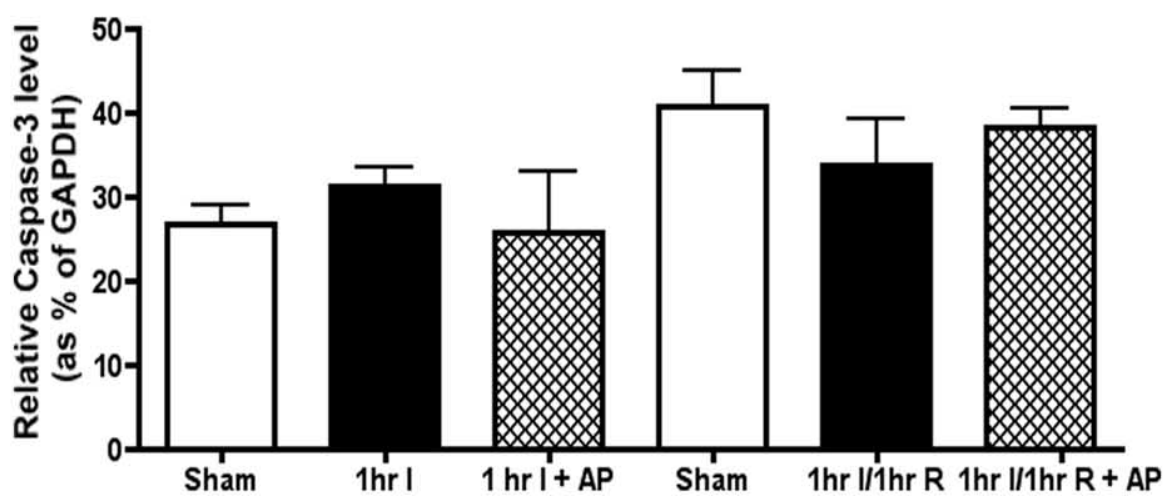

Figure 3. Active caspase protein expression in 1-hour sham controls (leftmost Sham), 1-hour ischemia with saline solution (1hr I), 1-hour ischemia with aprotinin (1hr I + AP), 2-hour sham controls (rightmost Sham), 1-hour ischemia and 1-hour reperfusion with saline solution $(1 \mathrm{hrl} / \mathrm{h} \mathrm{hr})$, and 1-hour ischemia and 1-hour reperfusion with aprotinin (1hrl/1hrR + AP). A, Representative immunoblot of caspase 8. B, Densitometric data of caspase 8 (as percentage of GAPDH). C, Representative immunoblot of caspase 3. D, Densitometric data of caspase 3 (as percentage of GAPDH). Bars represent mean; error bars indicate SEM. Asterisk indicates $\boldsymbol{P}<.01$ relative to sham controls; double asterisk indicates $P<.05$ relative to sham controls; delta indicates $P<.05$ relative to 1-hour ischemia and 1-hour reperfusion with saline solution. 

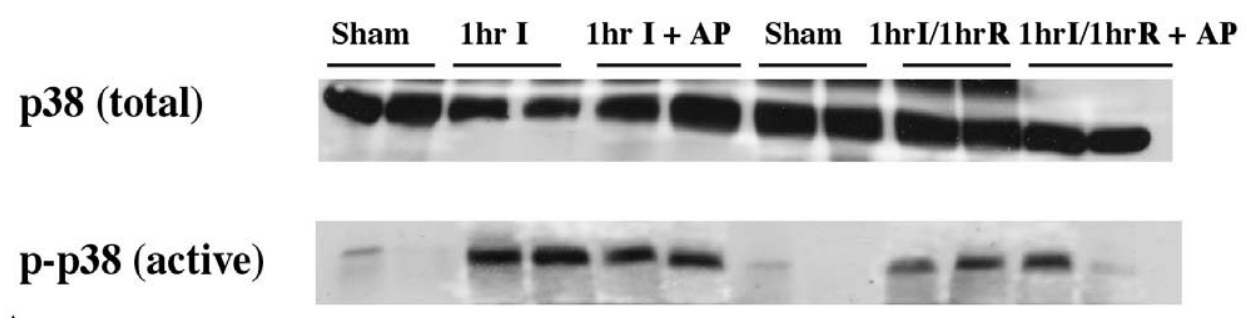

$\mathbf{A}$

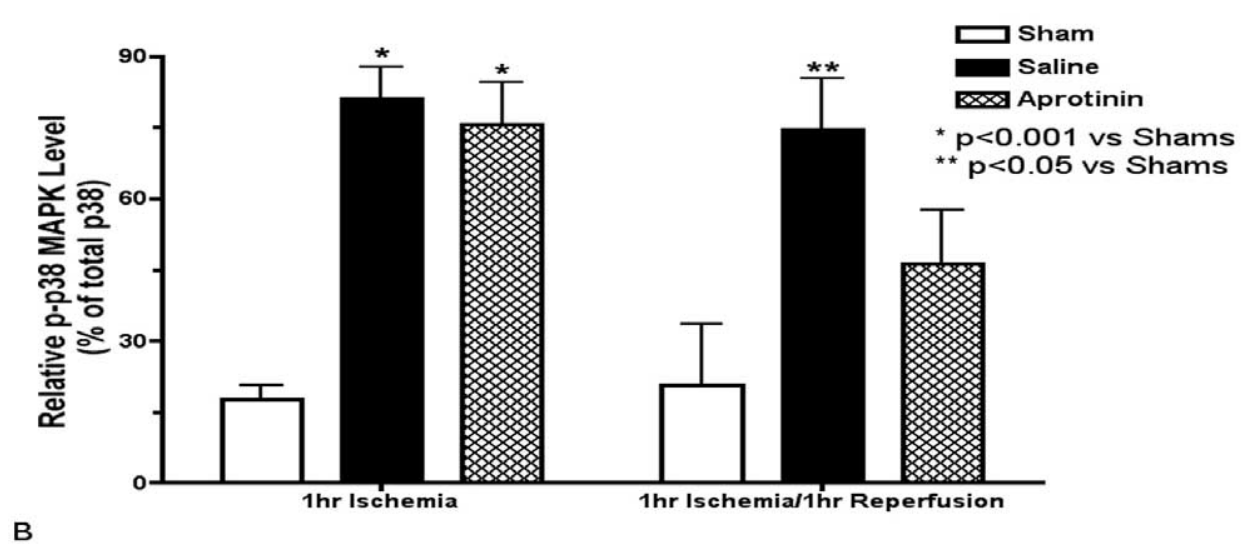

Figure 4. The expression of activated p38 MAPK in 1-hour sham controls (leftmost Sham), 1-hour ischemia with saline solution (1hr I), 1-hour ischemia with aprotinin (1hr I + AP), 2-hour sham controls (rightmost Sham), 1-hour ischemia and 1-hour reperfusion with saline solution (1hrl/1hrR), and 1-hour ischemia and 1-hour reperfusion with aprotinin (1hrl/1hrR + AP). A, Representative immunoblot. Top row shows nonphosphorylated p38 MAPK (total); bottom row shows phosphorylated p38 MAPK (activated). B, Densitometric data of phosphorylated p38 MAPK (percentage of total p38 MAPK). Bars represent mean; error bars indicate SEM. Asterisk indicates $\boldsymbol{P}<.001$ relative to sham controls; double asterisk indicates $P<.05$ relative to sham controls.

mediates its protective effect through some other mechanism. Renal ischemia-reperfusion causes endothelial activation and enhances endothelium-leukocyte interaction, resulting in leukocyte accumulation and injury. In addition, chemokines such as IL-8 and monocyte chemoattractant protein 1 are upregulated, which by attracting leukocytes may increase the injury produced. Aprotinin has been shown to affect leukocyte integrin expression and leukocyte extravasation and hence may provide protection in renal ischemia-reperfusion injury through this mechanism. Further studies looking at the impact of aprotinin on chemokines and leukocyte-endothelium interaction may help to clarify the mechanism of protection provided by aprotinin in renal ischemia-reperfusion.

Our results show that aprotinin decreases apoptosis and causes a significant reduction in caspase 8 , suggesting that the decrease in apoptosis may be mediated by altering the expression of caspase 8 . Caspase 3 is downstream to caspase 8, and we did not find any significant increase in its expression relative to sham controls, which may be because it is activated later. The importance in renal ischemia- reperfusion injury of p38 MAPK, an important mediator for both apoptosis and the production of proinflammatory cytokines, has been shown by the use of p38 MAPK inhibitors, which decrease TNF production and apoptosis and provide protection from renal ischemia-reperfusion injury. ${ }^{2,28}$ We have shown that aprotinin decreases p38 MAPK activation and thus may be responsible for the decreased proinflammatory cytokines and the decreased apoptosis. However, JNK and ERK, the two other MAPKs, have also been shown to increase after ischemia-reperfusion injury and play an important role in apoptosis. ${ }^{29,30}$ Evidence suggests that it may be the balance between p38/JNK and ERK that determines the fate of the cell after stress. Thus, the role of these MAPKs needs to be further evaluated to clarify the mechanism by which aprotinin acts and the relative importance of each in producing apoptosis in renal ischemiareperfusion. Effects of aprotinin on ischemia versus those on reperfusion indicate that aprotinin may impact ischemiareperfusion by at least two distinct mechanisms.

These results should be interpreted with certain caveats. As is already known, once renal injury reduces the number 

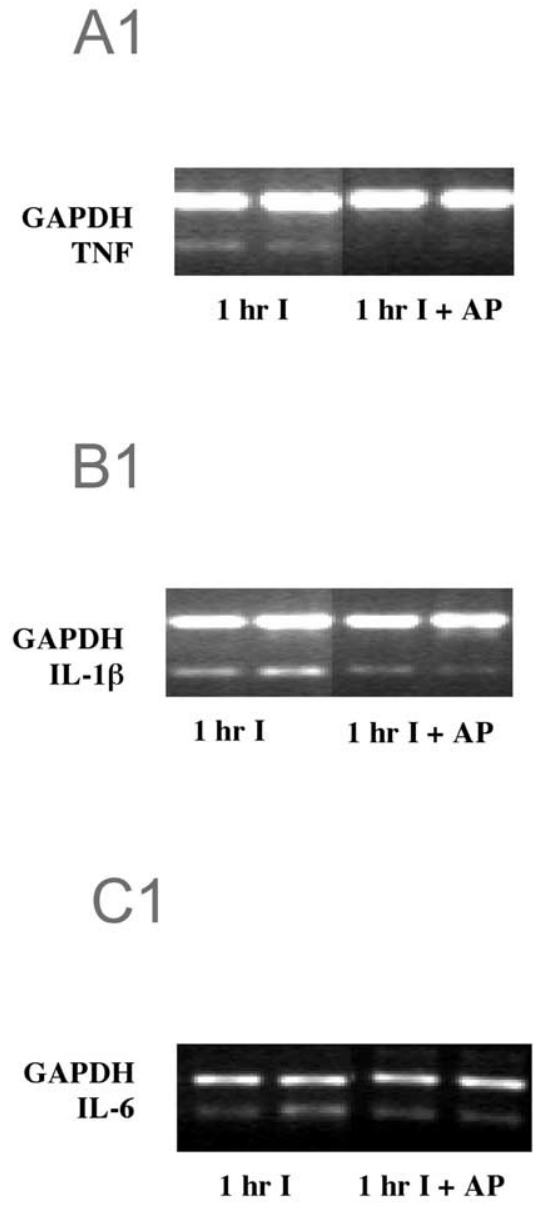
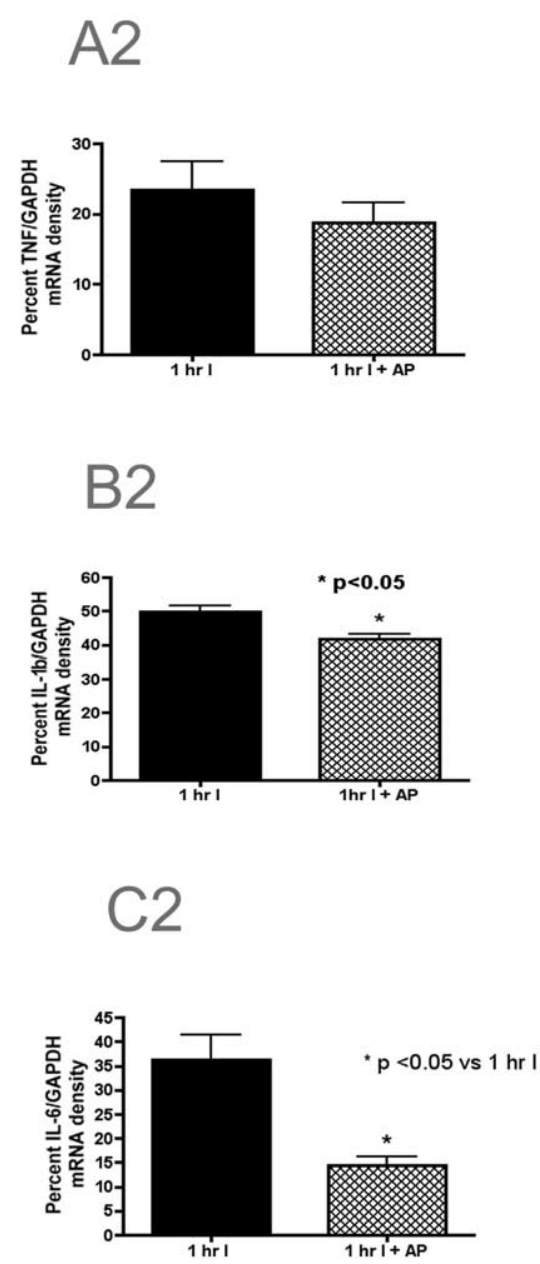

Figure 5. A1, B1, C1, Representative gel photographs depicting TNF- $\alpha$ (A1), IL-1 $\beta$ (B1), IL-6 (C1), and GAPDH RT-PCR bands in 1-hour ischemia with saline solution (1 hr I) and in 1-hour ischemia with aprotinin (1 hr I+ AP). A2, B2, C2, Densitometric data of TNF- $\alpha$ (A2), IL-1 $\beta$ (B2), and IL-6 (C2) are presented as percentage of GAPDH. Bars represent mean; error bars indicate SEM. Asterisk indicates $\boldsymbol{P}<.05$ relative to 1 -hour ischemia without aprotinin.

of functioning nephrons, chronic changes develop at an accelerated rate. Longer follow-up is therefore needed to see whether aprotinin is able to protect the nephrons from the development of these chronic changes. Our study used 1 hour of ischemia, and we studied inflammatory mediators at two time points. This provided data suggestive of a beneficial effect of aprotinin on renal ischemia-reperfusion injury. However, further studies that use different periods of ischemia and examine multiple time points for cytokine expression may help delineate the effects of aprotinin on renal inflammatory response to ischemia-reperfusion injury more clearly. Also, the use of inhibitors of p38 MAPK or antibodies to TNF may help clarify the role these play in mediating the protective effects of aprotinin on renal ischemia-reperfusion injury.

\section{References}

1. Meldrum KK, Burnett AL, Meng X, Misseri R, Shaw MB, Gearhart $\mathrm{JP}$, et al. Liposomal delivery of heat shock protein 72 into renal tubular cells blocks nuclear factor-kappaB activation, tumor necrosis factoralpha production, and subsequent ischemia-induced apoptosis. Circ Res. 2003;92:293-9.

2. Meldrum KK, Meldrum DR, Hile KL, Yerkes EB, Ayala A, Cain MP, et al. p38 MAPK mediates renal tubular cell TNF-alpha production and TNF-alpha-dependent apoptosis during simulated ischemia. Am J Physiol Cell Physiol. 2001;281:C563-70.

3. Donnahoo KK, Shames BD, Harken AH, Meldrum DR. The role of tumor necrosis factor in renal ischemia-reperfusion injury. $J$ Urol. 1999;162:196-203.

4. Donnahoo KK, Meng X, Ayala A, Cain MP, Harken AH, Meldrum DR. Early kidney TNF-alpha expression mediates neutrophil infiltration and injury after renal ischemia-reperfusion. Am J Physiol. 1999; 277:R922-9.

5. Donnahoo KK, Meng X, Ao L, Ayala A, Shames BD, Cain MP, et al. Differential cellular immunolocalization of renal tumour necrosis 
factor-alpha production during ischaemia versus endotoxaemia. Immunology. 2001;102:53-8.

6. Meldrum DR. Tumor necrosis factor in the heart. Am J Physiol. 1998;274:R577-95.

7. Meldrum KK, Meldrum DR, Meng X, Ao L, Harken AH. TNF-alphadependent bilateral renal injury is induced by unilateral renal ischemiareperfusion. Am J Physiol Heart Circ Physiol. 2002;282:H540-6.

8. Meldrum KK, Meldrum DR, Sezen SF, Crone JK, Burnett AL. Heat shock prevents simulated ischemia-induced apoptosis in renal tubular cells via a PKC-dependent mechanism. Am J Physiol Regul Integr Comp Physiol. 2001;281:R359-64.

9. Kher A, Wang M, Tsai BM, Pitcher JM, Greenbaum ES, Nagy RD, et al. Sex differences in the myocardial inflammatory response to acute injury. Shock. 2005;23:1-10.

10. Soeparwata R, Hartman AR, Frerichmann U, Stefano GB, Scheld HH, Bilfinger TV. Aprotinin diminishes inflammatory processes. Int J Cardiol. 1996;53 Suppl:S55-63.

11. Alonso A, Whitten CW, Hill GE. Pump prime only aprotinin inhibits cardiopulmonary bypass-induced neutrophil $\mathrm{CD} 11 \mathrm{~b}$ up-regulation. Ann Thorac Surg. 1999;67:392-5.

12. Asimakopoulos G, Kohn A, Stefanou DC, Haskard DO, Landis RC, Taylor KM. Leukocyte integrin expression in patients undergoing cardiopulmonary bypass. Ann Thorac Surg. 2000;69:1192-7.

13. Asimakopoulos G, Thompson R, Nourshargh S, Lidington EA, Mason JC, Ratnatunga CP, et al. An anti-inflammatory property of aprotinin detected at the level of leukocyte extravasation. J Thorac Cardiovasc Surg. 2000;120:361-9.

14. Kim KU, Kwon OJ, Jue DM. Pro-tumour necrosis factor cleavage enzyme in macrophage membrane/particulate. Immunology. 1993;80: 134-9.

15. Hill GE, Alonso A, Spurzem JR, Stammers AH, Robbins RA. Aprotinin and methylprednisolone equally blunt cardiopulmonary bypassinduced inflammation in humans. J Thorac Cardiovasc Surg. 1995; 110:1658-62.

16. Bull DA, Connors RC, Albanil A, Reid BB, Neumayer LA, Nelson R, et al. Aprotinin preserves myocardial biochemical function during cold storage through suppression of tumor necrosis factor. $J$ Thorac Cardiovasc Surg. 2000;119:242-50.

17. Diego RP, Mihalakakos PJ, Hexum TD, Hill GE. Methylprednisolone and full-dose aprotinin reduce reperfusion injury after cardiopulmonary bypass. J Cardiothorac Vasc Anesth. 1997;11:29-31.

18. Hill GE, Diego RP, Stammers AH, Huffman SM, Pohorecki R. Aprotinin enhances the endogenous release of interleukin-10 after cardiac operations. Ann Thorac Surg. 1998;65:66-9.
19. D'Ambra MN, Akins CW, Blackstone EH, Bonney SL, Cohn LH, Cosgrove DM, et al. Aprotinin in primary valve replacement and reconstruction: a multicenter, double-blind, placebo-controlled trial. J Thorac Cardiovasc Surg. 1996;112:1081-9.

20. Cosgrove DM 3rd, Heric B, Lytle BW, Taylor PC, Novoa R, Golding LA, et al. Aprotinin therapy for reoperative myocardial revascularization: a placebo-controlled study. Ann Thorac Surg. 1992;54:1031-8.

21. Lemmer JH Jr, Stanford W, Bonney SL, Chomka EV, Karp RB, Laub $\mathrm{GW}$, et al. Aprotinin for coronary artery bypass grafting: effect on postoperative renal function. Ann Thorac Surg. 1995;59:132-6.

22. Sedrakyan A, Treasure T, Elefteriades JA. Effect of aprotinin on clinical outcomes in coronary artery bypass graft surgery: a systematic review and meta-analysis of randomized clinical trials. $J$ Thorac Cardiovasc Surg. 2004;128:442-8.

23. Lemmer JH Jr, Dilling EW, Morton JR, Rich JB, Robicsek F, Bricker DL, et al. Aprotinin for primary coronary artery bypass grafting: a multicenter trial of three dose regimens. Ann Thorac Surg. 1996;62: $1659-68$

24. Smith PK, Muhlbaier LH. Aprotinin: safe and effective only with the full-dose regimen. Ann Thorac Surg. 1996;62:1575-7.

25. Schumer M, Colombel MC, Sawczuk IS, Gobe G, Connor J, O’'Toole $\mathrm{KM}$, et al. Morphologic, biochemical, and molecular evidence of apoptosis during the reperfusion phase after brief periods of renal ischemia. Am J Pathol. 1992;140:831-8.

26. Pruefer D, Buerke U, Khalil M, Dahm M, Darius H, Oelert H, et al. Cardioprotective effects of the serine protease inhibitor aprotinin after regional ischemia and reperfusion on the beating heart. $J$ Thorac Cardiovasc Surg. 2002;124:942-9.

27. Ozer Z, Sucu N, Dusmez D, Tamer L, Altunkan AA, DIkmengIl M, et al. The effect of aprotinin on ischemia-reperfusion injury in the rabbit kidney. Pharmacol Res. 2001;44:455-60.

28. Furuichi K, Wada T, Iwata Y, Sakai N, Yoshimoto K, Kobayashi Ki K, et al. Administration of FR167653, a new anti-inflammatory compound, prevents renal ischaemia/reperfusion injury in mice. Nephrol Dial Transplant. 2002;17:399-407.

29. Yin T, Sandhu G, Wolfgang CD, Burrier A, Webb RL, Rigel DF, et al. Tissue-specific pattern of stress kinase activation in ischemic/reperfused heart and kidney. J Biol Chem. 1997;272:19943-50.

30. Park KM, Chen A, Bonventre JV. Prevention of kidney ischemia/ reperfusion-induced functional injury and JNK, p38, and MAPK kinase activation by remote ischemic pretreatment. J Biol Chem. 2001;276:11870-6. 

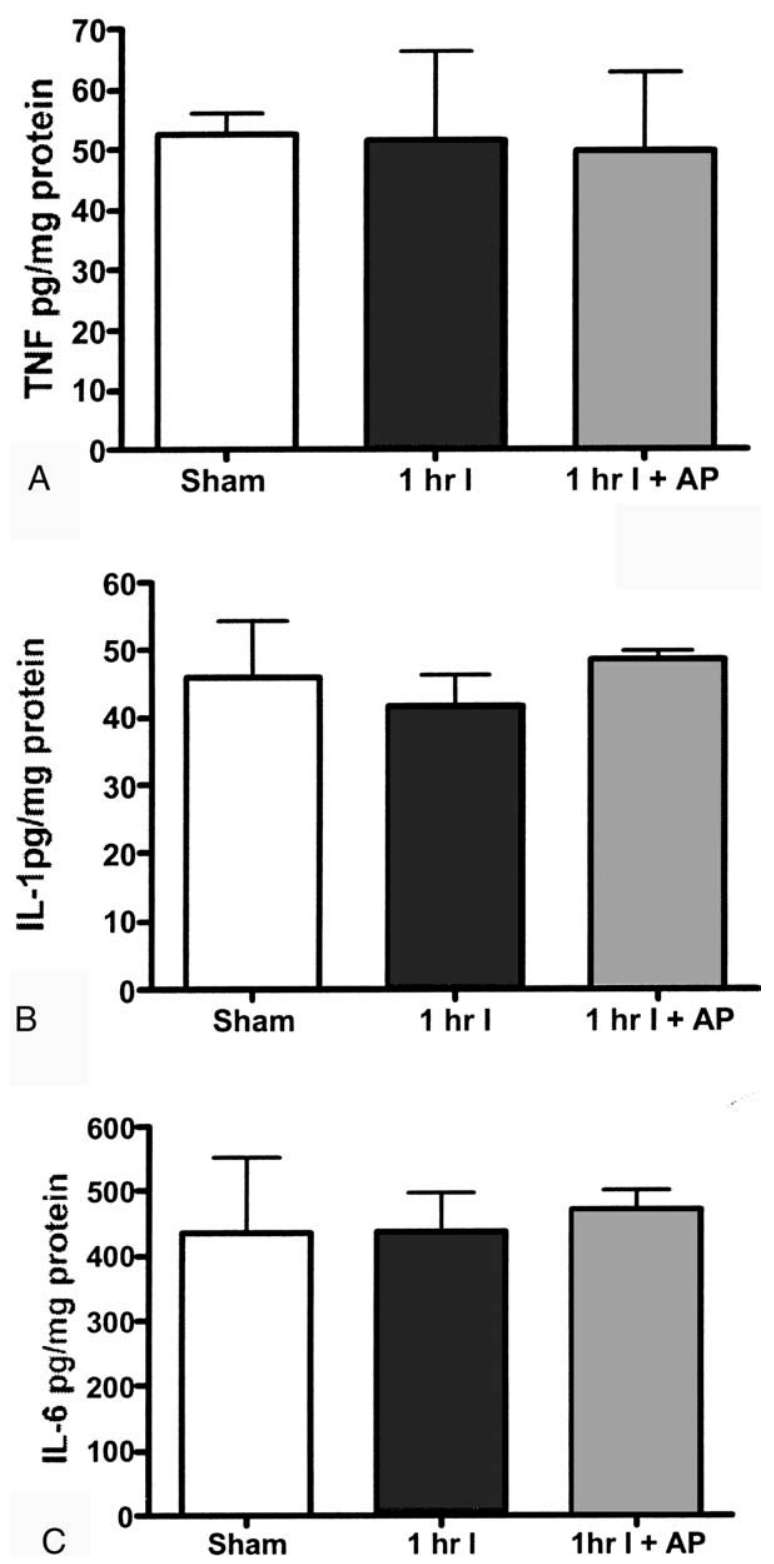

Figure E1. Renal TNF- $\alpha$ (A), IL-1 $\beta$ (B), and IL-6 (C) protein levels expressed as picograms of cytokine per milligram of protein in sham controls (Sham), 1-hour ischemia with saline solution (1 hr I), and 1-hour ischemia with aprotinin $(1 \mathrm{hr} I+A P)$. Bars represent mean; error bars indicate SEM. 


\section{A1}

GAPDH

TNF

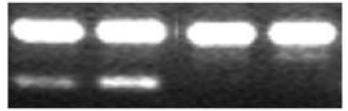

1hr I/1hr R 1 hr I/1hr R + AP

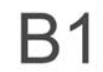

GAPDH

IL-1 $\beta$

1hr I/1hr R 1 hr I/1hr R + AP

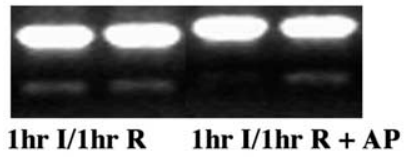

\section{C1}

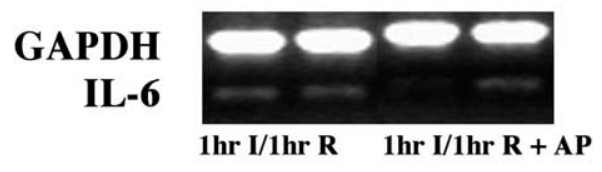

\section{A2}
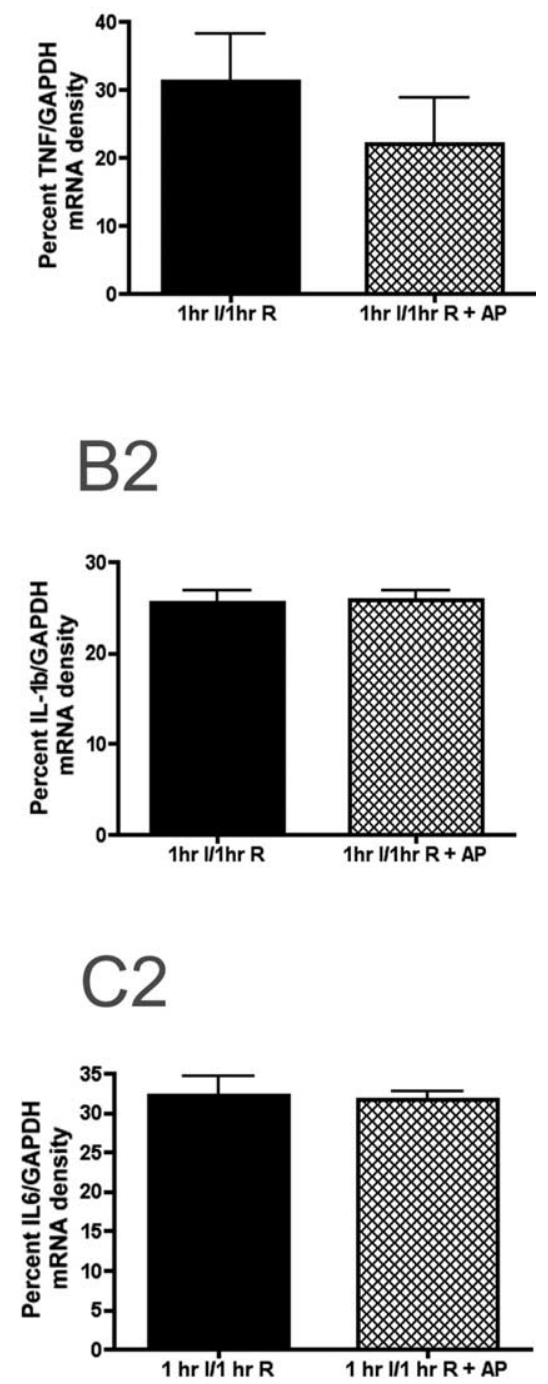

Figure E2. A1, B1, C1, Representative gel photographs depicting TNF- $\alpha$ (A1), IL-1 $\beta$ (B1), IL-6 (C1), and GAPDH RT-PCR bands in 1-hour ischemia and 1-hour reperfusion with saline solution (1 hr I/1 hr $R$ ) and in 1-hour ischemia and 1-hour reperfusion with aprotinin (1 hr l/ $1 \mathrm{hr} R+A P$ ). A2, B2, C2, Densitometric data of TNF- $\alpha$ (A2), IL-1 $\beta$ (B2), and IL-6 (C2) are presented as percentage of GAPDH. Bars represent mean; error bars indicate SEM. 
${ }^{\star *} p<0.01$
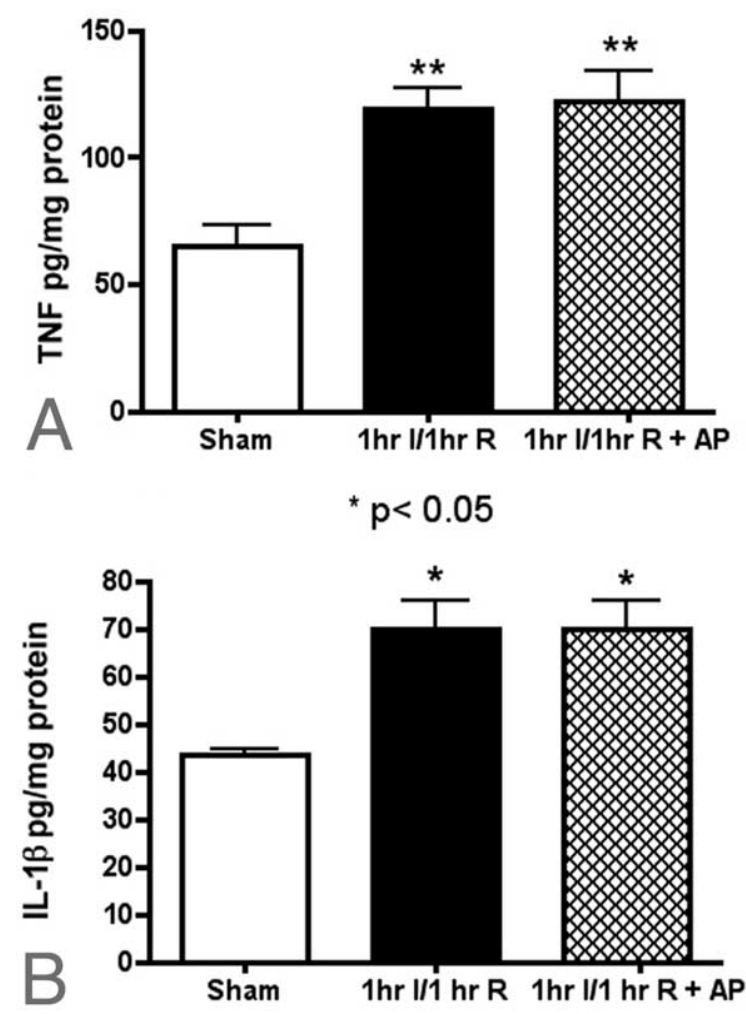

${ }^{*} p<0.05$

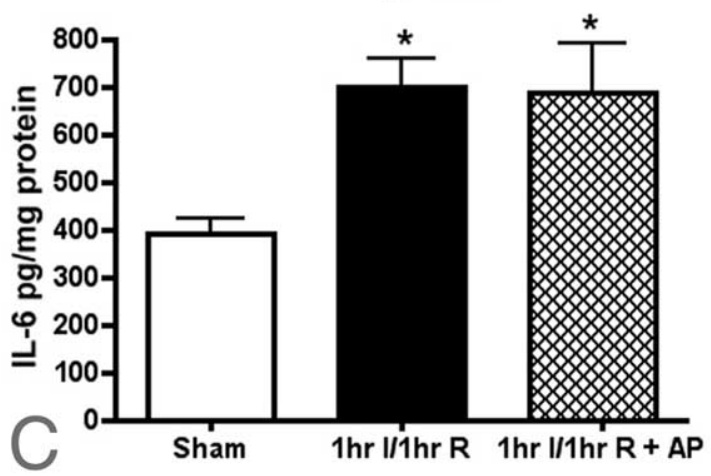

Figure E3. Renal TNF- $\alpha$ (A), IL-1 $\beta$ (B), and IL-6 (C) protein levels expressed as picograms of cytokine per milligram of protein in sham controls (Sham), 1-hour ischemia and 1-hour reperfusion with saline solution (1 hr I/1 hr R), and 1-hour ischemia and 1-hour reperfusion with aprotinin (1 hr l/ $1 \mathrm{hr} R+A P)$. Bars represent mean; error bars indicate SEM. Asterisk indicates $\boldsymbol{P}<.05$ relative to sham control; double asterisk indicates $\boldsymbol{P}<.01$ relative to sham control. 